\title{
SJOGREN'S SYNDROME WITH ACQUIRED HEMOPHILIA A: A CASE REPORT
}

Raíssa Dudienas Domingues Pereira ${ }^{1, \star}$, Vinícius Verlangieri Soubihe1, Diego de Paula Ferreira Nunes', Caio Rustichelli Cardoso', Zoraida Sachetto ${ }^{1}$

1.Universidade Estadual de Campinas, Campinas (SP), Brazil.

*Corresponding author: ra_dudienas@hotmail.com

\section{BACKGROUND}

Sjögren's syndrome is an autoimmune disease, characterized by lymphocytic infiltration, with preferential involvement of salivary and lacrimal glands, that can also lead to numerous extraglandular manifestations. Acquired hemophilia A is a rare pathology, difficult to diagnose, mainly due to its lack of knowledge. In this report, we describe a case of Sjögren's syndrome with acquired hemophilia A, diagnosed after hemorrhagic manifestation.

\section{CASE REPORT}

Female, 56 years old, being followed up with rheumatology because of the diagnosis of Sjögren's syndrome for about 1 year. In November 2019, she presented digestive hemorrhage (melena and enterorrhagia), leading to a significant drop in the level of serum hemoglobin, requiring hospitalization. In April 2020, right after venipuncture in the left arm, she started to experience local pain and the appearance of an extensive hematoma. After evaluation by the hematology team, which suggested the hypothesis of acquired hemophilia A, the presence of factor VIII inhibitor was evidenced, with a reduction of this factor, leading to the diagnosis of acquired hemophilia A. Due to this condition, with major bleeding, the infusion of activated prothrombin complex and immunosuppression with cyclophosphamide were indicated. After this, the patient progressively improved hematoma and arm pain. After immunosuppression, there was a drop in the levels of factor VIII inhibitor, without new hemorrhagic manifestations.

\section{CONCLUSION}

Acquired hemophilia $\mathrm{A}$ is a rare condition that, if not diagnosed quickly, has a high mortality rate. In the presence of a patient with autoimmune disease with hemorrhagic manifestations, its investigation should be carried out in order to avoid future complications. 